\title{
Measurement of the growth of the lateral ventricles in preterm infants with real-time ultrasound
}

\author{
MALCOLM I LEVENE \\ Department of Paediatrics and Neonatal Medicine, Institute of Child Health, Hammersmith Hospital, London
}

SUMMARY Real-time ultrasound was used to make exact measurements from the lateral wall of the body of the lateral ventricle to the falx (the ventricular index) in 273 infants of varying gestational ages. The measurement performed in an axial plane through the temporoparietal bone correlated closely with an actual measurement made in coronal plane in 50 infants. A cross-sectional centile chart was drawn up of the normal range for this measurement from 27 to 42 weeks' postmenstrual age. A further chart showing the rate of change of the ventricular index allowed growth of the ventricles to be assessed in a longitudinal manner. Use of these charts permits early detection of hydrocephalus or dilated ventricles secondary to cerebral atrophy.

Visualisation of the cerebral ventricles is essential in investigation of the child with suspected hydrocephalus. Four readily-available methods exist: echo encephalography and air ventriculography, and more recently computerised tomography and realtime ultrasound. Echo encephalography using A-mode equipment produces reliable measurements of midline structures such as the 3 rd ventricle, ${ }^{1-3}$ but measurement of lateral ventricles is more difficult and time consuming. ${ }^{4-6}$ Air encephalography is highly invasive and produces artefactual changes in lateral ventricular size, ${ }^{78}$ making its use severely limited.

The diagnosis of hydrocephalus on computerised tomography is often obvious, ${ }^{9}$ however little information is available on the normal range of ventricular size ${ }^{1011}$ and early hydrocephalus or dilated ventricles of mild degree may be missed. Fukuyama et al. ${ }^{12}$ gave a range for a ventricular ratio in normal children, although only 6 infants aged below 1 year were studied. Ventricular size has been measured prenatally in an effort to diagnose early fetal hydrocephalus, ${ }^{13}$ and postnatally during the first year of life ${ }^{414}{ }^{15}$ Studies have shown good correlation between the lateral ventricular ratio measured both by A-scan echo-encephalography and computerised tomography in infants. ${ }^{15} 16$

Recently, real-time ultrasound has been widely accepted as a reliable method to diagnose intraventricular haemorrhage and hydrocephalus in infancy. ${ }^{1718}$ There are as yet no data on the size of the ventricular systems in preterm infants, and for this reason a study of both cross-sectional and longitudinal ventricular growth was undertaken and centile charts constructed.

\section{Methods}

A Kranzbühler ADR linear array real-time ultrasound machine was used to visualise the body of the

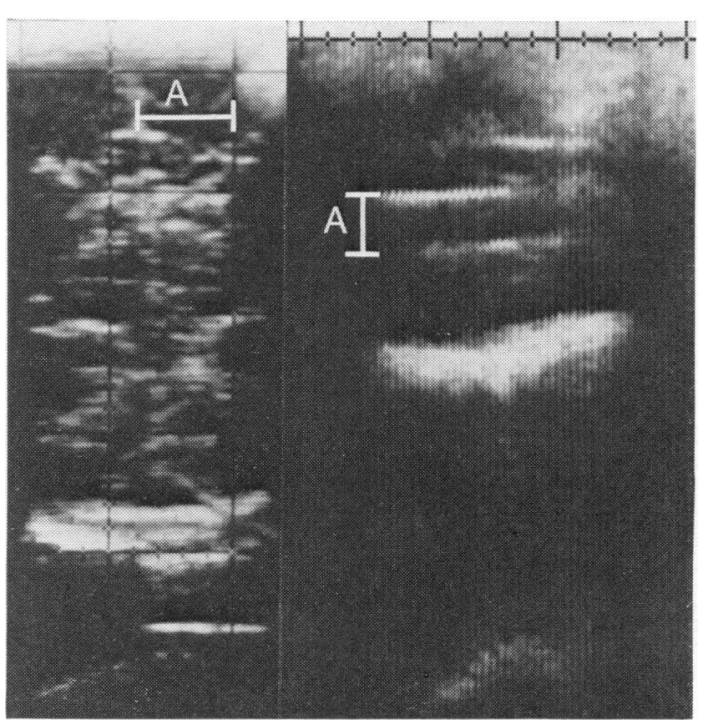

Fig. 1 (Right) axial scan (5 MHz transducer) through the temporoparietal bone showing the measurement from the midline echo to the lateral ventricle-the ventricular index $(A)$. (Left) coronal scan ( $7 \mathrm{MHz}$ transducer) through the fontanelle showing the measurement from the lateral-most extent of the lateral ventricle to the falx $(A)$. 
lateral ventricles in 273 term and preterm infants. Scanning was performed with a $5 \mathrm{MHz}$ transducer placed against the fairly flat temporoparietal region of the infant's head at the level of the outer canthus of the eye and the helix of the ear. ${ }^{18}$ The transducer is kept parallel to this line and is moved upwards until the body of the lateral ventricles is seen most clearly (Fig. 1). The distance between the echo from the falx and the contralateral ventricular wall is measured using omnidirectional calipers fitted to the machine. The system velocity was 1540 metres/second. Two measurements were made at each examination and averaged. As this is not a true measurement of the size of the lateral ventricle at this point, this distance is referred to as the ventricular index (VI). Serial measurements were performed on many infants until the skull had become sufficiently mineralised to make visualisation impossible, generally between ages 6 and 9 months.

Attenuation of ultrasound through the temporoparietal bone may cause distortion and make measurement of VI in this plane unreliable. For this reason the VI was compared with a transfontanelle coronal scan using a $7 \mathrm{MHz}$ transducer in 50 infants.
With this higher frequency transducer, the falx and ventricul $r$ system could be clearly visualised, and an actual measurement made from the falx to the most lateral extent of the lateral ventricle (Fig. 1).

Healy's method of estimating the mean and standard deviation (SD) by 'censoring' the lowest and highest $5 \%$ of the measurements for each gestational age group was used. ${ }^{19}$ The change in VI from birth to the end of the second week of life was calculated and expressed as a velocity $(\mathrm{mm} / 2$ weeks). This was repeated for successive 2-week intervals until the infant was 28 weeks old, irrespective of gestation. The mean and SDs of each velocity measurement during a 2-week interval was calculated using this method. In order to detect any variation in ventricular size occurring before the end of the second week of life, the change in VI at age 7 days was calculated, as was the mean and SD.

\section{Subjects}

The infants studied ranged from 26 to 42 weeks' gestation. Some $(n=50)$ were normal term infants (gestational age 37 to 42 weeks) examined on the

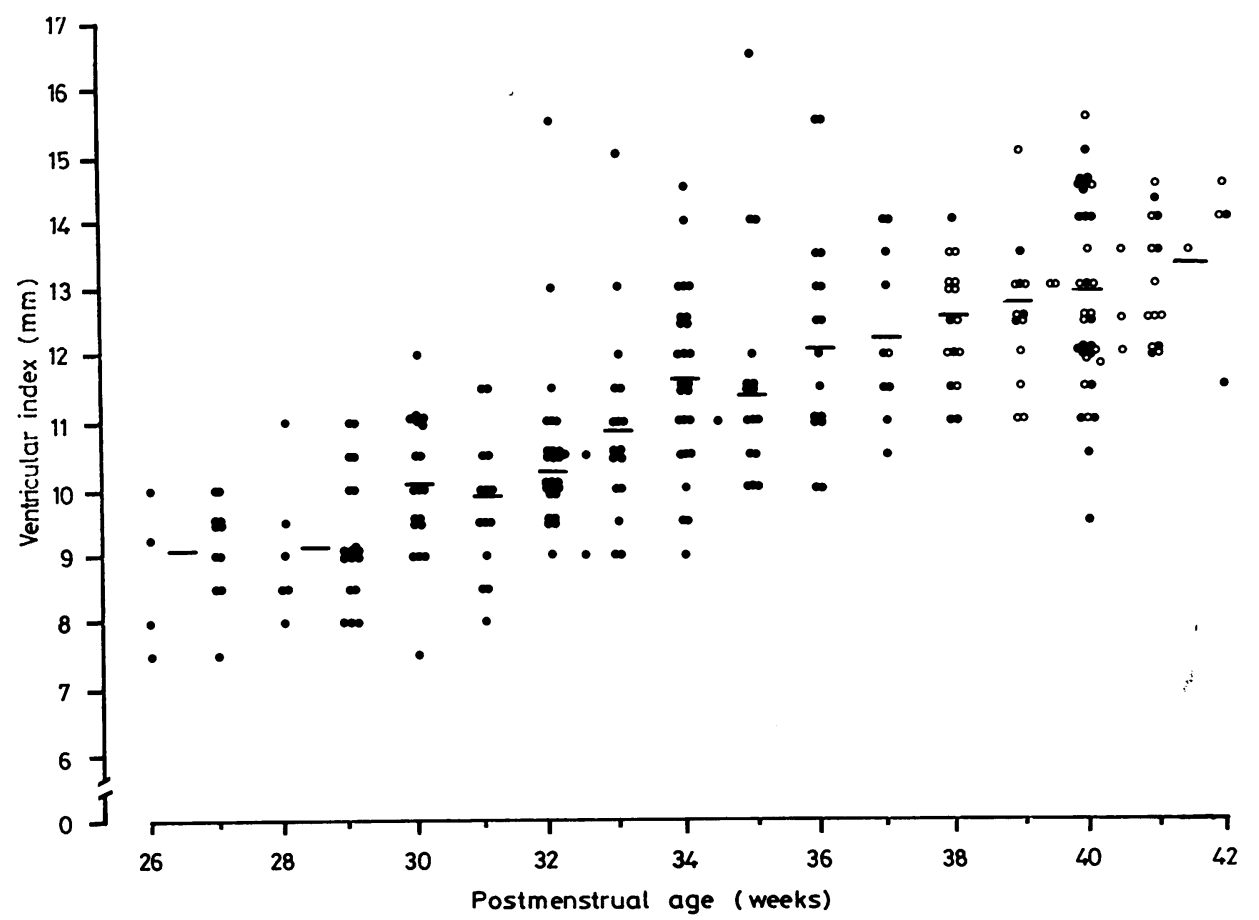

Fig. 2 Distribution of ventricular index by gestational age. Open circles represent 'normal' infants examined on the postnatal ward. The mean is shown by a horizontal bar. Closed circles represent infants admitted to the neonatal unit for prematurity or clinical problems. 
postnatal ward after informed parental consent. The remaining 223 infants had been admitted to a neonatal unit for observation or treatment. The first scan was performed within the first 6 days of life, and in $81 \%$ of them was within 48 hours of birth. If an infant had ultrasound evidence of intraventricular haemorrhage or cerebral atrophy on the first scan he was excluded from the study; of the 273 infants, only 8 subsequently developed intraventricular haemorrhage after an initially normal scan. Fig. 2 shows the distribution of infants by gestational age and their VI measurements. Thirtythree babies died during the course of the study and $90(48 \%)$ of the survivors returned to the follow-up clinic and were subsequently rescanned at each visit and measurements of VI made.

\section{Results}

The mean and SD were calculated weekly as described from 30 to 40 weeks of gestational age. Results for 26 and 27, 28 and 29, and 41 and 42 were considered together because of the small numbers (Fig. 2). Fig. 3 shows the relationship between the actual measurement on coronal scan from the lateral-most extent of the lateral ventricle to the falx and the VI measured through the temporoparietal bone $(r=$ 0.98). A cross-sectional centile chart of the VI shows the smoothed 3rd, 50th, and 97th centiles for this measurement over the gestational ages 26 to 42 weeks (Fig. 4). Fig. 5 shows the smoothed growth velocity during 2-week intervals from birth to 28 weeks' chronological age, irrespective of gestation.

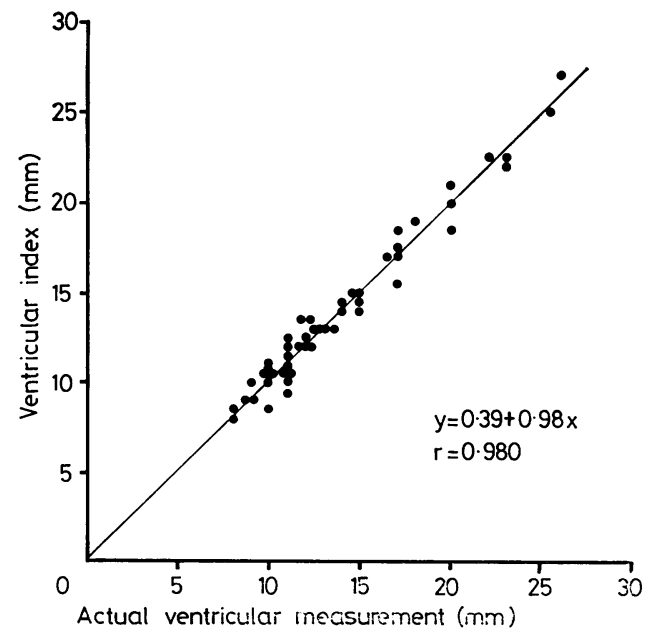

Fig. 3 Correlation of ventricular index $(\mathrm{mm})$ with actual ventricular measurement $(\mathrm{mm})$.

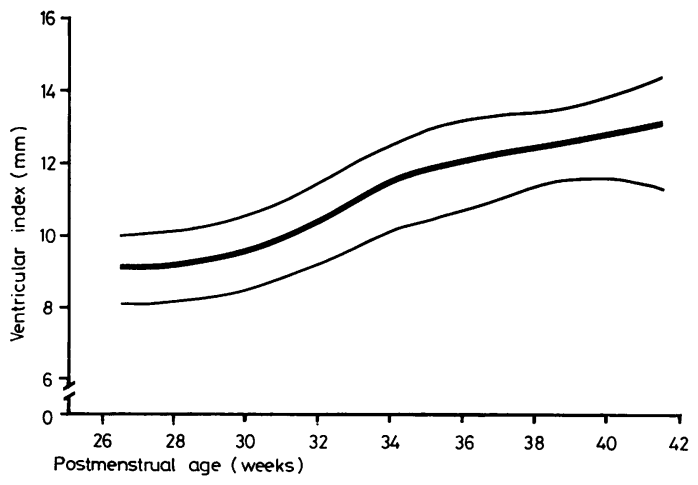

Fig. 4 Cross-sectional chart of ventricular index. Smoothed centiles are 3rd, 50th, and 97th.

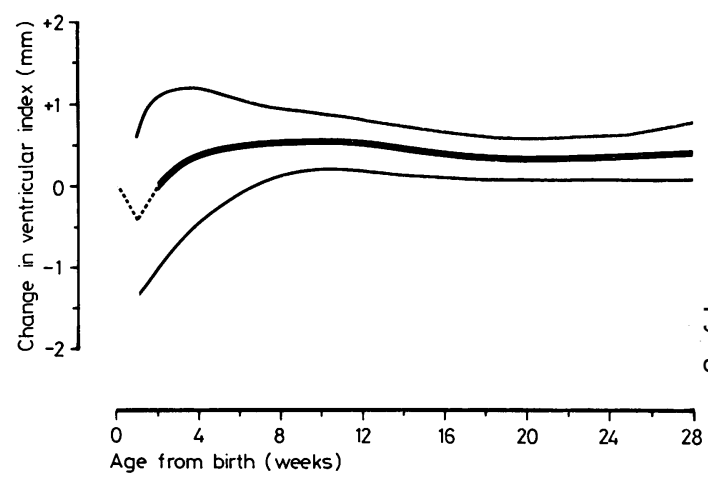

Fig. 5 Chart showing growth velocity from age 2 weeks. Dotted lines represent change in ventricular index during the first and second weeks of life. Smoothed centiles are 3rd, 50th, and 97th.

The broken line represents the change in VI from birth to the end of the first week, and from 7 days to the end of the second week. A comparison between mean growth rates in less mature babies (less than or equal to 33 weeks) and those of 34 weeks or more is shown in Fig. 6. During the first 6 months of life there is no significant difference between the mean values for the first 10 weeks and from the 20th week of life. Between 12 and 18 weeks, the rate of increase of the VI in the less mature babies is slightly higher than in the more mature ones and the difference is significant during each of those 6 weeks $(P<0 \cdot 05)$.

Infants were measured three times during the course of an hour (by MIL) and the VI did not vary by more than $1 \mathrm{~mm}$. Inter-observer error depended on the experience of the observer. The Table shows two estimates of VI measurements on 10 infants 

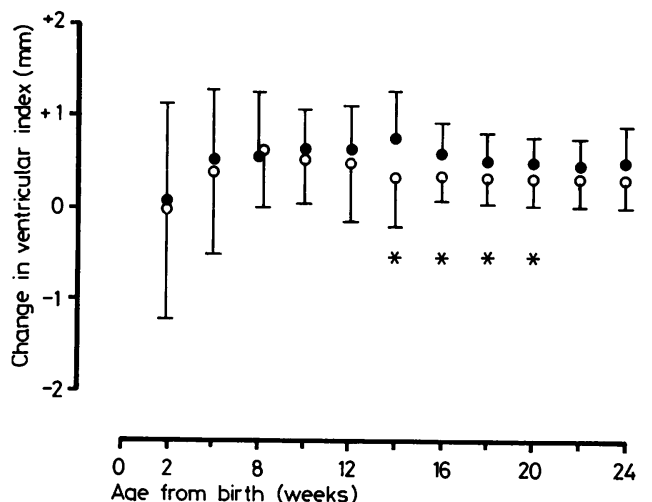

Fig. 6 Comparison of the mean $( \pm S D)$ for change in ventricular index in preterm infants 33 weeks and below (closed circles) with more mature infants of 34 weeks and over (open circles). Asterisk represents a significant difference between the means $(P<0.05)$.

Table Estimate of inter-observer error by 2 experienced ultrasonographers

\begin{tabular}{lcc}
\hline Case & $\begin{array}{l}\text { First observer } \\
(M I L)\end{array}$ & $\begin{array}{l}\text { Second observer } \\
(M R L)\end{array}$ \\
\hline 1 & 9,9 & 9,9 \\
2 & 9,9 & 9,9 \\
3 & 11,11 & 11,11 \\
4 & 10,10 & 10,9 \\
5 & 8,9 & 9,9 \\
6 & 10,10 & 10,9 \\
7 & 11,12 & 11,11 \\
8 & 12,12 & 12,12 \\
9 & 11,12 & 12,12 \\
10 & 12,11 & 11,11 \\
\hline
\end{tabular}

performed at different times on the same day by two experienced observers (MIL and MRL), each with more than 6 months' scanning time.

\section{Discussion}

Use of real-time ultrasound provides a safe, rapid, and convenient method to measure the size of the lateral ventricles. In order to assess the true prevalence of dilated ventricles, enlargement beyond a normal range must be shown. This study provides such a range of ventricular size for various gestational ages, thus allowing those infants in whom this measurement is more than 2 SDs above the mean to be identified. Longitudinal growth velocity charts for ventricular size permit rate of change in ventricular size in an individual infant to be closely monitored in order to detect abnormal rates of enlargement.

This description provides ventricular growth data in only one direction but measurements in other positions are difficult. The shape of the cerebral ventricles is complicated and changes with brain maturation. To monitor changes in size of this system, one or a number of reference points must be used. These should be readily identifiable, should be constant in position, and should not vary in shape between patients. The occipital poles of the lateral ventricles are generally the largest part of the entire ventricular system, however no convenient landmark exists and there may be considerable variation in shape of this structure. ${ }^{20}$ The body of the lateral ventricle is easily accessible with ultrasound through the anterior fontanelle and is symmetrical in shape and position. There is a clear maximum distance between the most lateral extent of the ventricle and the falx which can rapidly be measured. Changes in size of the body of the lateral ventricle appear closely to mirror changes within the entire lateral ventricular system, and such changes can be closely monitored.

Although the measurements may be made rapidly and agree well between the same observer and different observers, experience is necessary before consistent reproducibility is achieved. A new investigative technique requires practice but reliable measurements should be obtained in a short time by a user familiar with ultrasound methods.

In the past, the measurement of the lateral ventricular size has usually been expressed as a ratio to the skull diameter. Evans ${ }^{7}$ introduced the much used encephalographic ratio for estimating the size of the cerebral ventricles in air-encephalography and this has subsequently been used in computerised tomography scanning. ${ }^{21} \mathrm{~A}$ modification of it has been adopted in ultrasound measurements of ventricular size in infants. ${ }^{1522}$ More recently, doubt has been cast on the value of the Evans's ratio and direct measurement of the ventricular size recommended..$^{23}$ In this study, the very close correlation between the actual measurement of ventricular size made on coronal section and the ventricular index permits the VI to be used as a valid measurement of true ventricular size and makes unnecessary the use of a ratio which is more limited in terms of monitoring growth.

Change in the VI in the neonate shows a clear pattern which does not vary to any great extent with the maturity of the infant. There is initially a reduction in size of the lateral ventricular measurement during the first week of life. Significant shrinkage of head size during the first week in infants weighing less than $2 \mathrm{~kg}$ has been reported, ${ }^{24}$ and in addition, a reduction in cerebral blood flow ${ }^{25}$ and fluctuations in intracranial pressure ${ }^{26}$ also occur during the first few days of life. The precise reason for these changes is not clear, but a reduction in size of the lateral ventricles during this time is not unexpected. The most rapid increase of the VI occurs from the end 
of the first week to the sixth week of life and thereafter the rate is slower.

Real-time ultrasound provides a method for measuring lateral ventricular size in infants. Measurement of the VI allows diagnosis of dilated ventricles for a given gestational age, and regular assessment of size permits early detection of deviation in lateral ventricular growth. Use of these charts permits both hydrocephalus and cerebral atrophy to be diagnosed early in their development and appropriate action taken.

I thank Dr M R Lebed for assistance, Miss A Smith for secretarial help, Professor Victor Dubowitz for help and encouragement, and the Department of Medical Art, Royal Postgraduate Medical School, for the illustrations.

This work was supported by the Wellcome Trust.

\section{References}

1 Grumme T, Frömmel G, Meese W. Post natale echoencephalographische Untersuchungen bei Frühgeborenen. Neuropaediatrie 1971; 3: 155-70.

2 Grumme T, Meese W, Frömmel G. Echoencephalographische kontrolluntersuchungen an Frühgeborenen während der ersten 18 Lebensmonate. Neuropaediatrie 1974; 5: 243-9.

3 Grumme T. Die Breite der 3 Hirnkammer vom Frühgeborenen bis ins 10 Dezennium. Fortschr Neurol Psychiatr 1977; 45: 223-68.

- Dill R. Echoencephalographische Normalwerte des kindlichen Ventrikelsystems in den vershiedenen Altersgruppen. Monatsschr Kinderheilkd 1971 ; 119: 496-502.

5 Wealthall S R, Todd J H, Ball C. The role of A-scope encephalography in childhood hydrocephalus. Dev Med Child Neurol 1973; Supplement 29, 92-9.

- Tenner M S, Wodraska G, Adapon B D. Newer ultrasound techniques in the evaluation of neurologic disorders. Radiol Clin N Am 1974; 12: 283-95.

7 Evans W A, Jr. An encephalographic ratio for estimating ventricular enlargement and cerebral atrophy. Arch Neurol Psychiatr 1942; 47: 931-7.

8 Olgaard A. Changes in ventricular size during pneumoencephalography. Neuroradiology 1971; 3: 8-11.

9 Naidich T P, Epstein F, Lin J P, Kricheff I I, Hochwald G M. Evaluation of pediatric hydrocephalus by computed tomography. Radiology 1976; 119: 337-45.

10 Hahn F J Y, Rim K. Frontal ventricular dimensions on normal computed tomography. AJR 1976; 126: 593-6.
11 Wolpert S M. The ventricular size on computed tomography. J Comput Ass Tomogr 1977; 1: 222-6.

12 Fukuyama $Y$, Miyao M, Ishizu T, Maruyama $H$. Developmental changes in normal cranial measurements by computed tomography. Dev Med Child Neurol 1979; 21: 425-32.

13 Denkhaus $\mathrm{H}$, Winsberg F. Ultrasonic measurement of the fetal ventricular system. Radiology $1979 ; 131$ : 781-7.

14 Lombroso C T, Erba G, Yogo T, Logowitz N. Twodimensional ultrasonography: a method to study normal and abnormal ventricles. Pediatrics 1968; 42: 157-74.

15 Johnson M L, Mack L A, Rumack C M, Frost M, Rashbaum C. B-mode echoencephalography in the normal and high risk infant. AJR 1979; 133: 375-81.

16 Hanson J, Levander B, Liliequist B. Size of the intracerebral ventricles as measured with computer tomography, encephalography, and echoventriculography. Acta Radiol [Suppl] (Stockh) 1975; 346: 98-106.

17 Pape K E, Blackwell R J, Cusick G, et al. Ultrasound detection of brain damage in preterm infants. Lancet 1979; i: $1261-4$.

18 Levene M I, Wigglesworth J S, Dubowitz V. Cerebral structure and intraventricular haemorrhage in the neonate: a real-time ultrasound study. Arch Dis Child 1981; 56: 416-24.

19 Healy M J R. A mean difference estimator of standard deviation in symmetrically censored normal samples. Biometrika 1978; 65: 643-6.

20 Last R J, Tompsett D H. Casts of the cerebral ventricles. Br J Surg 1953; 40: 525-43.

21 Gyldensted C. Measurements of the normal ventricular system and hemispheric sulci of 100 adults with computed tomography. Neuroradiology 1977; 14: 183-92.

22 Johnson M L, Rumack C M. Ultrasonic evaluation of the neonatal brain. Radiol Clin N Am 1980; 18: 117-31.

23 Zatz L M. The Evans ratio for ventricular size: a calculaze tion error. Neuroradiology 1979; 18: 81.

24 Williams J, Hirsch N J, Corbet A J S, Rudolph A J. Postnatal head shrinkage in small infants. Pediatrics 1977; 59: 619-22.

25 Cooke R W I, Rolfe P, Howat P. Apparent cerebral blood-flow in newborns with respiratory disease. Dev Med Child Neurol 1979; 21: 154-60.

26 Donn S M, Philip A G S. Early increase in intracranial pressure in preterm infants. Pediatrics 1978; 61: 904-7.

Correspondence to Dr M I Levene, Department of Paediatrics and Neonatal Medicine, Institute of Child Health, Hammersmith Hospital, Du Cane Road, London W12 0HS.

Received 30 June 1981 